\title{
Paradigma pragmático-funcional en la rehabilitación de la afasia
}

\section{Carlos Hernández Sacristán \\ Universidad de Valencia}

Facultad de Filología, Traducción y

Comunicación

Departamento de Teoría de los Lenguajes y

Ciencias de la Comunicación

Avda. Blasco Ibáñez 32

46010 Valencia

E-mail: carlos.hernandez-sacristan@uv.es

\author{
Vicent Rosell Clari \\ Universidad de Valencia \\ Facultad de Psicologia \\ Departamento de Psicología Básica \\ Universidad de Valencia \\ Avda. Blasco Ibáñez, 21 \\ 46010 Valencia \\ E-mail: vicente.rosell@uv.es
}

\section{PARADIGMA PRAGMÁTICO-FUNCIO- NAL EN LA REHABILITACIÓN DE LA AFASIA}

RESUMEN: Durante las últimas dos décadas, la investigación sobre la rehabilitación de la afasia ha desarrollado una visión pragmática y funcional del lenguaje, lo que ha llevado a una orientación cada vez más multifacética de trabajo terapéutico. En este enfoque pragmático-funcional de la rehabilitación de la afasia convergen diferentes programas concebidos de forma independiente, centrando la terapia en la dimensión relacional del lenguaje, que in cluye: las interrelaciones funcionales entre los componentes del sistema lingüístico; la función comunicativa $\mathrm{y}$ e uso del lenguaje en contextos naturales como las conversaciones cotidianas; las interrelaciones entre el comportamiento verbal y otros medios semióticos como la gestualidad; y las interrelaciones entre el lenguaje y otras habilidades cognitivas. Estos precedentes pueden ser considerados en conjunto para esbozar un nuevo paradigma para la rehabilitación de la afasia. Las dimensiones básicas de este paradigma y la relación jerárquica entre las mismas, son presentadas en el presente estudio.

PALABRAS CLAVES: rehabilitación afasia; conversación; metacognición; funcionalismo.

SUMARIO: 1. Introducción. 2. Sentido y alcance de una orientación pragmático-funcional. 2.1. Funcionalidad como factor constitutivo del sistema lingüistico. 2.2. Funcionalidad y comunicación. 2.3. Funcionalidad y actividad metacognitiva. 2.4. Otros aspectos relacionados con la funcionalidad: intensidad del tratamiento y transferibilidad de resultados. 3. Paradigma pragmático-funcional como plan heurístico para la terapia de la afasia. 4. Conclusiones.

\section{PRAGMATIC-FUNCTIONAL PARA- DIGM IN APHASIA REHABILITA- TION}

ABSTRACT: Over the last two decades, research on aphasia rehabilitation has developed a pragmatic, functional view of language, leading to an increasingly multi-faceted orientation of therapeutic work. Different programmes, independently conceived, converge in this pragmatic-functional approach to aphasia rehabilitation, to focus therapy on the relational dimension of language, which includes: functional interrelationships between components of the linguistic system; communicative function and the use of language in natural contexts as everyday conversations; interrelationships between verbal behaviour and other semiotic means such as gesturing; and interrelationships between language and other cognitive abilities These precedents can be considered together to outline a new paradigm for aphasia rehabilitation. The basic dimensions of this paradigm, and the hierarchical relationship between them, are presented in this study.

KEY WORDS: rehabilitation, aphasia; conversation; metacognition; functionalism.

SUMMARY: 1. Introduction. 2. Sense and scope of a pragmatic-functional orientation. 2.1. Functionality as a constituent factor of the language system. 2.2. Functionality and communication. 2.3. Functionality and metacognitive activity. 2.4. Other aspects related to functionality: intensity of treatment and transferability of results. 3. Pragmatic-functional paradigm as heuristic plan for aphasia therapy. 4. Conclusions.

\section{PARADIGME FONCTIONNEI PRAGMATIQUE DANS REHABILITATION DE L'APHASIE}

RÉSUMÉ: Au cours des deux dernières décennies, la recherche sur la réhabilitation de l'aphasie a développé une vision pragmatique et fonctionnelle $\mathrm{du}$ langage, conduisant à une orientation de plus en plus multidimensionnelle ${ }^{\text {plus }}$ du travail thérapeutique. Différents programmes de conception indépendante se rencontrent dans cette approche pragmatique-fonctionnel de la réhabilitation de l'aphasie, pour centrer la thérapie sur la dimension relationnelle du langage, qui comprend: les interrelations fonctionnelles entre les constituants du système linguistique; la fonction communicationnelle et l'utilisation du langage dans un contexte nature comme la pratique des conversations quotidiennes; les relations entre la conduite verbale et les autres moyens sémiotiques comme les gestes et les rapports entre le langage et les autres capacités cognitives. Ces précédents peuvent être considérés ensemble afin de tracer un nouveau paradigme pour la réhabilitation de l'aphasie. Les dimensions fondamentales de ce paradigme, et la relation hiérarchique entre elles, sont présentées dans cette étude.

MOTS CLÉS: réhabilitation; aphasie; conversation; métacognition fonctionnalisme.

SOMMAIRE: 1. Introduction. 2. Sens et portée d'une orientation pragmatiquefonctionnelle. 2.1. La fonctionnalité en tant que facteur constitutif du système linguistique. 2.2. Fonctionnalité et communication. 2.3. Fonctionnalité et activité métacognitive. 2.4. Autres aspects liés à la fonctionnalité : intensité du traitement et transférabilité des résultats. 3. Paradigme pragmatiquefonctionnel comme plan heuristique pour la thérapie de l'aphasie. 4 Conclusions.
Fecha de Recepción Fecha de Revisión Fecha de Aceptación Fecha de Publicación 


\title{
Paradigma pragmático-funcional en la rehabilitación de la afasia $^{1}$
}

\author{
CARlos HeRnÁndeZ SACRISTÁn \& Vicent Rosell Clari
}

\section{INTRODUCCIÓN}

La revisión de los protocolos de rehabilitación de la afasia de las últimas dos décadas nos muestra una escena realmente compleja. Aparentemente, tanto los supuestos teóricos que sustentan las tareas de rehabilitación, como los criterios técnicos para la evaluación de los programas de rehabilitación son de naturaleza muy heterogénea. La American Speech-LanguageHearing Association (ASHA) ofrece, por ejemplo, una lista de programas de rehabilitación de la afasia muy variada en cuanto a la concepción y alcance de los mismos (https: / /www.asha.org/practice-portal/clinical-topics/aphasia/). En algunos casos los programas se centran en dominios lingüísticos específicos, como la sintaxis o las unidades léxicas. En otros, los programas no están orientados a dominios particulares de organización del sistema lingüístico, sino que se refieren a la conducta lingüística y/o comunicativa del paciente en general.

En algunos casos los beneficios terapéuticos de los componentes semióticos no verbales, como la gestualidad o la entonación, constituyen el centro de atención. Pero también encontramos algún programa terapéutico, en concreto el llamado Constraint Induced Language Therapy (CILT) (cf. para una versión actualizada Johnson et al., 2014), que descarta explícitamente estos componentes al asumir su efecto negativo sobre la recuperación de la conducta verbal. Existen además algunos programas definidos por el instrumento específico utilizado para la rehabilitación, como es el caso de los tratamientos basados en medios informáticos, o por la teoría lingüística específica que sustenta el programa de rehabilitación. Algunos programas se concentran en la práctica lingüística individual del paciente, mientras que otros se centran en la interacción comunicativa del paciente con sus interlocutores clave. El alcance de los factores contextuales incluidos en los programas terapéuticos también es muy variado.

Los programas pueden ser en algún aspecto contradictorios, pero en general observamos solapamientos o maneras de proceder que podrian considerarse complementarias. En cualquier caso, el terapeuta del lenguaje puede quedar perplejo con la variedad de propuestas disponibles. Ante ese panorama de posibles acciones terapéuticas que se ofrecen, parece razonable, antes de sumar otra opción más a la lista, proponer lo que podría denominarse un plan heurístico sobre los factores implicados en los programas

\footnotetext{
${ }^{1}$ Este estudio forma parte de las actividades financiadas por el proyecto ministerial FFI201784951-P: Dimensiones metacognitivas en la adquisición, las alteraciones clínicas del lenguaje y la práctica interlingüística. Un enfoque pluridisciplinar.
} 
de rehabilitación de la afasia, para facilitar así, de alguna manera, las decisiones del logopeda relativas a la elección del mejor tratamiento para casos individuales, o de la mejor combinación o secuencia de tratamientos. La combinación de programas de terapia puede ser contraproducente cuando se aplica de forma descontextualizada y sin tener en cuenta la idiosincrasia del paciente, pero quizás no cuando se administran con el mismo objetivo terapéutico que es mejorar la capacidad comunicativa del paciente en su entorno.

Ofrecer un plan heurístico supone retrotraerse hacia un punto desde donde tomar una perspectiva panorámica que evite esa circunstancia comúnmente descrita de que "los árboles no nos dejan ver el bosque". Sin esta visión panorámica es imposible trazar la ruta que permita seleccionar de manera crítica entre las acciones terapéuticas que se ofrecen para dar solución a los casos particulares de intervención. En el presente estudio este plan heurístico al que nos referimos se articula en términos de un "paradigma pragmático-funcional" para la rehabilitación de la afasia (extensible a otro tipo de patologías centrales del lenguaje) (Rosell-Clari y HernándezSacristán, 2017, 2018b; Hernández Sacristán, 2019). En nuestra propuesta se tienen en cuenta las contribuciones recientes al tema, formuladas desde diferentes perspectivas y posiciones teóricas, pero que comparten de manera más o menos explícita una visión común sobre la naturaleza del lenguaje y sobre los factores implicados en su recuperación después de un accidente cerebrovascular.

El objetivo es proporcionar un marco conceptual integrado que nos permita visualizar las conexiones y los puntos en común, así como contribuir a resolver las contradicciones aparentes entre las diferentes propuestas terapéuticas. Una orientación funcional en el estudio de la conducta verbal significa considerar el lenguaje como un fenómeno relacional complejo, que incluye tanto la organización interna del sistema lingüístico como el entorno cognitivo y sociocultural del uso del lenguaje. Un punto de vista funcional sobre el lenguaje implica considerar esta facultad humana como un producto complejo de interdependencias sobre las que debe normalmente trabajar el logopeda.

La búsqueda de este tipo de integración conceptual de los programas terapéuticos para la afasia debería, en nuestra opinión, ir acompañada de una búsqueda de métodos y criterios de investigación unificadores. Este último objetivo está claramente justificado cuando se observa también la inconsistencia y el carácter no conclusivo de los procedimientos para la evaluación de la eficacia de los programas terapéuticos. Brady et al. (2014) se hacen eco en un "consensus paper" de esta problemática y apuestan por una profunda labor de meta-análisis que permita comparar los resultados de investigaciones previas. También en este caso lo que se plantea no es sumar una nueva investigación más a la lista, basada normalmente en casos únicos o con un número limitado de sujetos, sino realizar análisis comparativos de 
estudios previos, esto es, meta-análisis que suponen una notable ampliación del número de sujetos implicados a la hora de fundamentar la eficacia de las propuestas de rehabilitación. En términos de los mencionados autores:

In the recent Cochrane systematic review and synthesis of 39 randomised controlled trials of speech and language therapy for people with aphasia, it became evident that there was room for more methodologically robust trial designs (Brady et al., 2012). Where data were available, trials typically randomised small numbers of participants (mean 65 (SD 68) range 5-327 individuals) from very diverse populations in terms of age (range 17-97 years), time since stroke (mean of 3.2 days up to 28 years) and aphasia severity (mild to very severe). A priori sample size calculations were only reported in $15 \%$ $(6 / 39)$ of included trials. Therapy regimes were many and varied, and there was little consistency in the outcomes measured (Brady et al., 2012) (Brady et al., 2014: 1387)

Es obvio, por otra parte, que esta labor comparativa requiere también según Brady et al. (2014: 1386) acuerdos previos sobre la manera en que deben reflejarse los resultados de investigación, algo de lo que se sigue estando muy lejos, según se afirma en Ali et al. (2013) y Salter et al. (2007) (apud Brady et al., 2014: 1386). En cualquier caso, tras la inspección de conjunto a la que se refieren Brady et al. (2014), si hay un posible denominador común en las propuestas de investigación sobre la intervención en la afasia, este denominador se expresa en los términos propios de una orientación pragmático-funcional:

A key overlap in CDEs shared across aphasia rehabilitation research and trials of effectiveness is the common goal of enhancing (and thus the need to accurately measure) functional communication ability in real-world settings or activities. This agenda, which is shared by patients, family members, therapists and researchers, will facilitate a common language to describe "recovery", regardless of the nature of the intervention (Brady et al., 2014: 1387).

Esta conclusión relativa a la actividad investigadora no deja de ser congruente con los objetivos genéricos del tratamiento de la afasia que propone la ASHA, que potencian una terapia centrada en la persona y en sus interacciones sociales (http://www.asha.org/uploadedFiles/ICF-Aphasia.pdf)

\section{SENTIDO Y ALCANCE DE UNA ORIENTACIÓN PRAGMÁTICO-FUNCIONAL}

Como trataremos de esclarecer en lo que sigue, hablamos del binomio pragmático-funcional, entendiendo que lo "funcional" asume, pero al mismo tiempo rebasa el ámbito estricto de organización interna del sistema lingüístico, proyectándonos sobre una amplia gama de factores contextuales que condicionan y orientan el uso del lenguaje (cf. Gallardo Paúls y Hernández Sacristán, 2013). Para ello resulta crítico asumir, de entrada, la naturaleza relacional de los hechos del lenguaje, con la que se evita una visión atomizadora relativa a tareas concretas o micro-procesos cognitivos asociados a las mismas. 


\subsection{FUNCIONALIDAD COMO FACTOR CONSTITUTIVO DEL SISTEMA LINGÜÍSTICO}

Aunque pueda considerarse razonable un objetivo terapéutico inicial que se centre en déficits lingüísticos particulares, está claro que cualquier dominio de la capacidad lingüística, como por ejemplo el del acceso a las unidades léxicas, no puede concebirse nunca como una tarea estrictamente independiente de otras, lo que tiene claras implicaciones para la intervención logopédica. Por seguir con el ejemplo citado, la capacidad de recuperar una palabra particular en situaciones de anomia depende de la capacidad de recuperar otras palabras relacionadas con ella semántica o formalmente, o de la capacidad de recuperar palabras que frecuentemente se combinan con ella. Para trabajar en la recuperación de un determinado elemento léxico normalmente se requieren actividades que involucran elementos léxicos pertenecientes al entorno lingüístico funcional inmediato de este elemento.

Este componente relacional es explotado en propuestas concretas como la de denominación inducida por circunloquios (Francis et al., 2002) o en otras propuestas terapéuticas de la anomia que focalizan diferentes componentes relacionales de las unidades léxicas como las presentadas por McNeil et al. (1995) o Spencer et al. (2000). Se asume en general el hecho de que puede ser más eficaz focalizar la intervención en el entorno funcional de la unidad objeto de rehabilitación que en la propia unidad considerada de manera descontextualizada. En este sentido, y como proponen Helm-Estabrooks y Albert $(1991,2003)$, las unidades conservadas dentro de un determinado dominio léxico pueden ser utilizadas como punto de partida o "trampolin" para la recuperación de otras unidades que resultan circunstancialmente inaccesibles. Este procedimiento puede utilizarse como regla general para otros dominios del sistema lingüístico, como por ejemplo las construcciones sintácticas o las unidades fonológicas. El proceder al que nos referimos se corresponde con el primer nivel de funcionalidad lingüistica que sometemos aquí a consideración: una unidad lingüística debe siempre entenderse como constituida teniendo en cuenta su entorno relacional con otras unidades lingüisticas. La operatividad terapéutica propia de este nivel funcional puede sintetizarse con la fórmula "lenguaje rehabilita lenguaje", esto es, usamos el lenguaje para rehabilitar el propio lenguaje. Las capacidades verbales remanentes serian instrumentos que deben activarse para la recuperación de los recursos perdidos. Esta formulación encuentra de alguna manera su correlato en la adquisición y desarrollo de la capacidad lingüística. También el uso mismo del lenguaje es, según Tomasello (2003), la base para el aprendizaje de la primera lengua: "el uso del lenguaje crea el lenguaje". 


\subsection{FUNCIONALIDAD Y COMUNICACIÓN}

En un segundo nivel, la funcionalidad tiene que ver con la relación entre el lenguaje y la actividad comunicativa. La existencia de un objetivo comunicativo dota al lenguaje de funcionalidad en un sentido muy básico, que afecta a la naturaleza misma de su procesamiento cognitivo. Nos referimos al modo procedimental (frente al declarativo) que domina en el uso común del lenguaje, y con el que las propias estructuras lingüísticas se van configurando. El procesamiento cognitivo procedimental implica en el caso del lenguaje un desplazamiento del foco atencional hacia un objetivo comunicativo que trasciende al propio uso lingüístico, esto es, que dota al uso de "intencionalidad". Es obvio que en las sesiones terapéuticas resulta inevitable enfrentar al paciente con materiales lingüisticos descontextualizados, esto es, no intencionalmente guiados por objetivos comunicativos concretos, pero tal vez existen maneras de simular o evocar situaciones de uso comunicativo real, que deberán proponerse en lo posible, como se sugiere en el test MetAphAs (Rosell-Clari y Hernández-Sacristán, 2014, 2018a) y en la manera de proceder que se sigue en Rosell-Clari y Hernández-Sacristán (2017, 2018b).

El lenguaje, en definitiva, no puede ser aislado del propósito comunicativo para el que ha sido concebido. Con todo, cierto grado de disociación entre eficacia comunicativa y recursos lingüísticos disponibles puede ser postulado. Como en el caso extremo descrito por Goodwin (1995, 2000), la comunicación puede ser de alguna forma preservada en hablantes afásicos no fluentes con muy pocos recursos lingüisticos disponibles. La ayuda de los interlocutores y de otros medios semióticos no lingüísticos puede servir para este fin y compensar el déficit lingüístico específico en diferentes grados. Por el contrario, la preservación de competencias lingüísticas específicas no siempre garantiza -como es bien sabido- una comunicación eficaz en la vida cotidiana. Sin embargo, la observación de estas situaciones anómalas de disociación entre lo comunicativo y lo lingüístico, no debe llevarnos a concluir erróneamente que las habilidades lingüisticas puedan ser consideradas como independientes de su función comunicativa. De hecho, los recursos lingüísticos remanentes en los casos de afasia no fluente amplian el alcance de su significado en la medida en que son interpretados y utilizados como medio para lograr una pluralidad de objetivos comunicativos. Por lo tanto, estos recursos ven maximizado su potencial semiótico cuando se relacionan funcionalmente con diferentes contextos comunicativos. Por otro lado, también debe decirse que tener a nuestra disposición recursos lingüísticos que no pueden ser interpretados y utilizados para lograr objetivos comunicativos, como sucedería en muchos casos de logorrea, es lo mismo que disponer de un texto escrito en un alfabeto no descifrado. La facultad del lenguaje no puede ser identificada con el dominio de formas puramente positivas, privadas de un componente relacional y consideradas fuera de su uso particular para fines comunicativos. 
En efecto, no es fácil distinguir entre los trastornos lingüísticos y de comunicación cuando, de hecho, se considera el lenguaje desde un punto de vista funcional, como propuso inicialmente Halliday (1978) y otros muchos autores más adelante. Los contextos sociales y la pragmática forman parte del sistema lingüístico desde el punto de vista de Halliday. Si esto último se admite, en la labor del logopeda se requerirá un tratamiento dual de los trastornos tanto lingüisticos como comunicativos, sin que exista una precedencia previamente establecida de los unos sobre los otros. La reparación del instrumento lingüístico puede ser en algunas circunstancias un paso necesario para mejorar la comunicación, pero en otras circunstancias una intervención que desarrolle las habilidades comunicativas puede ser la mejor manera de crear el contexto para la recuperación del instrumento lingüístico. Para reforzar este punto de vista, consideremos también que las lesiones cerebrales que causan afasia generalmente producen otras anomalias asociadas que involucran las funciones cognitivas y motoras del individuo afectado y, en consecuencia, su interacción social y sus dimensiones emocionales y motivacionales. Todo esto nos lleva a considerar la afasia no sólo como una forma de deficiencia del lenguaje, sino como un trastorno de la comunicación más general.

La comunicación en un entorno natural es una actividad articulada de manera compleja. Los medios verbales se suelen combinar en toda comunicación natural con medios semióticos no verbales, que incluyen la gestualidad, la situación comunicativa y los referentes. Lo que se dice depende de cómo se orquestan los diferentes estratos de la comunicación no verbal. En la adquisición de la primera lengua los medios comunicativos no verbales proporcionan, de hecho, la matriz dentro de la cual se desarrollan las habilidades lingüisticas específicas. Esta dependencia metalingüística del lenguaje con respecto al contexto comunicativo permanece siempre operativa.

Como regla general, un déficit primario en los medios lingüísticos suele estar asociado a un déficit comunicativo, y los grados en los que se restringe la comunicación pueden ser considerados como una medida del déficit lingüístico, es decir, el déficit lingüístico debe ser evaluado teniendo en cuenta la gravedad de sus efectos sobre la práctica comunicativa. Este punto de vista ha sido comúnmente asumido en la investigación sobre la terapia del habla y del lenguaje para las personas con afasia. Podemos referirnos aquí a los trabajos pioneros de Holland (1979, 1980) y Sarno (1969), que destacaban como objetivo terapéutico antes la "comunicación funcional" que la "precisión lingüística" para las personas con afasia. En esta linea han sido otros muchos los autores que han sometido a exploración con fines clínicos las habilidades comunicativas de los pacientes en contextos naturales. Armstrong y Ferguson (2010) proponen, por ejemplo, una exploración del uso multifacético del lenguaje en contexto, con el objetivo de introducir claves para la mejora de sus interacciones comunicativas diarias. Sandt-Koenderman et al. (2012) identifican una perspectiva funcional en su revisión de terapias de la afasia, que tiene por objeto desarrollar un nivel óptimo de 
comunicación teniendo en cuenta el déficit lingüístico existente. Junto a ella proponen también una perspectiva participativa (también funcional en un sentido amplio de lo funcional en el que aquí estamos interesados), que se centra en estimular la integración social y eliminar las barreras que la impiden. Esta visión funcional global ha recibido un desarrollo especial en programas que se centran en la actividad conversacional (Kagan et. al., 2001; Simmons-Mackie et al., 2010; Carter et al., 2010; Wilkinson y Wielaert, 2012; Johnson et al., 2014).

Se reconoce de manera general que la eficacia en la terapia del lenguaje requiere de la participación activa del individuo afectado y, sin lugar a dudas, la actividad conversacional representa el contexto más propicio para ello. El uso del lenguaje en la conversación afecta al hablante en todas sus dimensiones cognitivas, personales e incluso somáticas. En el contexto propio de la práctica conversacional el hablante está continuamente obligado a tomar decisiones con el fin de utilizar el lenguaje de forma estratégica. Estas decisiones y las estrategias adoptadas están en gran medida motivadas por la imagen social que ponemos en juego cuando participamos en una conversación. El uso del lenguaje en la conversación está condicionado y al mismo tiempo motivado por el hecho de que normalmente tratamos de proteger o mejorar nuestra imagen social cuando hablamos. Así se explica, por ejemplo, que el uso de prolongados turnos conversacionales en pacientes afásicos con problemas de comprensión se haya interpretado como una manera de evitar la posición de oyente, dadas las dificultades que manifiestan cuando asumen este último rol (cf. Perkins, 2000: 21).

La dimensión somática del hablante también está presente en la conversación. Aquí podemos incluir marcadores emocionales asociados con la producción y comprensión del lenguaje, y medios semióticos no verbales como el gesto fónico y kinésico que acompañan a la actividad verbal y que se han considerado instrumentos complementarios en la rehabilitación de la afasia (Rose y Douglas, 2003; Rose, 2006).

Además, la proyección intersubjetiva del lenguaje se manifiesta especialmente en la conversación. Nuestras contribuciones lingüísticas están condicionadas por las inferencias que normalmente hacemos sobre los conocimientos previos, las expectativas o los estados mentales de nuestros interlocutores. De esta manera, la práctica conversacional siempre requiere una actividad de teoría de la mente (Rosell-Clari y Valles-González, 2016). Muchos programas terapéuticos potencian el papel desempeñado por los interlocutores clave, cuidadores o familiares, en su interacción comunicativa con personas con afasia (Holland, 1991; Kagan, 1998). Está claro que las terapias basadas en la conversación se centran en diferentes dimensiones de la naturaleza relacional del lenguaje y deben caracterizarse en un sentido pleno como "funcionales". 


\subsection{FUNCIONALIDAD Y ACTIVIDAD METACOGNITIVA}

Se puede también postular -y esto seria ya un tercer nivel de las relaciones funcionales que afectan al lenguaje- que esta facultad se encuentra relacionada con otros procesos o habilidades cognitivas, como la inhibición, la atención, la memoria, la planificación y otras funciones ejecutivas. El déficit lingüístico afecta en diferentes grados a las funciones ejecutivas, dado que el lenguaje es hasta cierto punto un instrumento implicado en el funcionamiento ejecutivo. En el modelo de funcionamiento ejecutivo propuesto por Barkley (1997) el "habla interior" es un componente que regula la atención humana, la memoria y los procesos emocionales. La actividad propia de la memoria de trabajo está claramente ligada al lenguaje interior, pero en general todo tipo de conductas y experiencias humanas están determinadas en gran medida por el lenguaje.

Por otro lado, el lenguaje, en sí mismo, es también una conducta regulada por las funciones ejecutivas. Este es el caso, en particular, del uso conversacional del lenguaje. Como acaba de señalarse en la sección precedente, la práctica conversacional estimula el uso estratégico del lenguaje y, por lo tanto, la interrelación entre conducta verbal y funciones ejecutivas. La inhibición, la atención, la memoria de trabajo, la planificación del comportamiento y el control emocional son funciones ejecutivas dialécticamente interrelacionadas con la actividad lingüística, lo que puede observarse paradigmáticamente en la práctica conversacional (véase al respecto Frankely Penn, 2007; Frankel et al., 2007; Penn et al., 2010). De hecho, el lenguaje aparece aquí por doble partida, en tanto objeto de estos procesos metacognitivos y en tanto que instrumento que participa activamente en el control metacognitivo de otras conductas humanas. Es por ello que desde la ASHA se considera la afasia como un trastorno cognitivo-comunicativo.

Siendo este el caso, la terapia del lenguaje no puede (y no debe) disociarse de la intervención en el dominio metacognitivo del funcionamiento ejecutivo. La rehabilitación de las habilidades lingüísticas manifestadas en la conversación y en otras prácticas naturales del lenguaje implica la intervención en el dominio metacognitivo que determina la conducta verbal. Este dominio incluye también, de manera muy significativa, la actividad reflexiva o metalingüistica sobre la propia conducta verbal, actividad que se evalúa en el protocolo MetAphAs (Metalanguage in AphasiaAssessment) (Hernández-Sacristán et al., 2012; Rosell-Clari y Hernández-Sacristán, 2014, 2018). En definitiva, un trabajo centrado en las funciones ejecutivas puede ser considerado como una forma de mejorar las habilidades lingüísticas y también cabe decir, a la inversa, que la terapia del lenguaje implicará normalmente una mejora en el funcionamiento ejecutivo general. 


\subsection{OTROS ASPECTOS RELACIONADOS CON LA FUNCIONALIDAD: INTENSIDAD DEL TRATAMIENTO Y TRANSFERIBILIDAD DE RESULTADOS}

Brady et al. (2012) revisaron los efectos de la Terapia del Habla y del Lenguaje (SLT: Speech and LanguageTherapy) en una muestra de 1,840 pacientes con afasia que recibieron tratamiento en los 30 años anteriores al estudio. Los resultados de esta revisión indican que existen pruebas concluyentes sobre la efectividad de la SLT para las personas con afasia. También se observó que la SLT intensiva proporciona mejores resultados que la SLT convencional. Según Johnson et al. (2014), las terapias que han demostrado su eficacia en la rehabilitación de personas con afasia crónica comparten lo siguiente: (a) la intensidad de la terapia; (b) la participación del cuidador o de personas importantes en la vida del paciente, y (c) la concentración en la resolución de problemas y la participación en las tareas comunicativas diarias, en lugar de un tratamiento exclusivamente clínico. La intensidad de la terapia es un factor comúnmente mencionado cuando se explica la eficacia del tratamiento. Este factor merece una breve consideración. Las bases cognitivas y fisiológicas para los procesos de aprendizaje o reaprendizaje son suficientes para explicar su relevancia, pero, en nuestros términos, la intensidad de la terapia contribuye especificamente a la puesta en juego activa de los componentes relacionales implicados en la recuperación del lenguaje.

La transferencia de los logros obtenidos en las sesiones clínicas a la práctica comunicativa diaria puede considerarse el objetivo último de la terapia del lenguaje y la prueba real de la eficacia de esta última. Nuestro objetivo terapéutico es restablecer, en la medida de lo posible, las habilidades lingüísticas y comunicativas del paciente vinculadas a las interacciones sociales pre-mórbidas, garantizando al menos una capacidad básica de interacción comunicativa en la vida cotidiana. En nuestra opinión, sólo las terapias que mejoran los componentes relacionales generales del lenguaje están diseñadas adecuadamente para transferir eficazmente los logros de los pacientes en las sesiones de rehabilitación a sus situaciones comunicativas comunes.

\section{PARAdigma PRAGMÁtico-FunCIONAL COMO PlAN HEURÍstico PARA LA TERA- PIA DE LA AFASIA}

Las perspectivas comentadas en la sección anterior y los factores a los que se suele hacer referencia como esenciales para la eficacia de un programa de terapia del lenguaje pueden considerarse en su conjunto bajo el prisma de un paradigma pragmático-funcional. Estos factores han sido evaluados individualmente como herramientas terapéuticas, pero nada impide trabajar con ellos de manera conjunta. Cada uno de ellos, individualmente, mejora los componentes relacionales del lenguaje involucrados como factores críticos en la terapia del lenguaje, pero refuerzan sus efectos cuando se 
ponen en juego de manera coordinada dentro de las sesiones de terapia. A continuación esbozaremos los componentes básicos que definen el paradigma pragmático-funcional, reformulando en parte lo contenido en Hernández-Sacristán (2019) y Rosell-Clari y Hernández-Sacristán (2017).

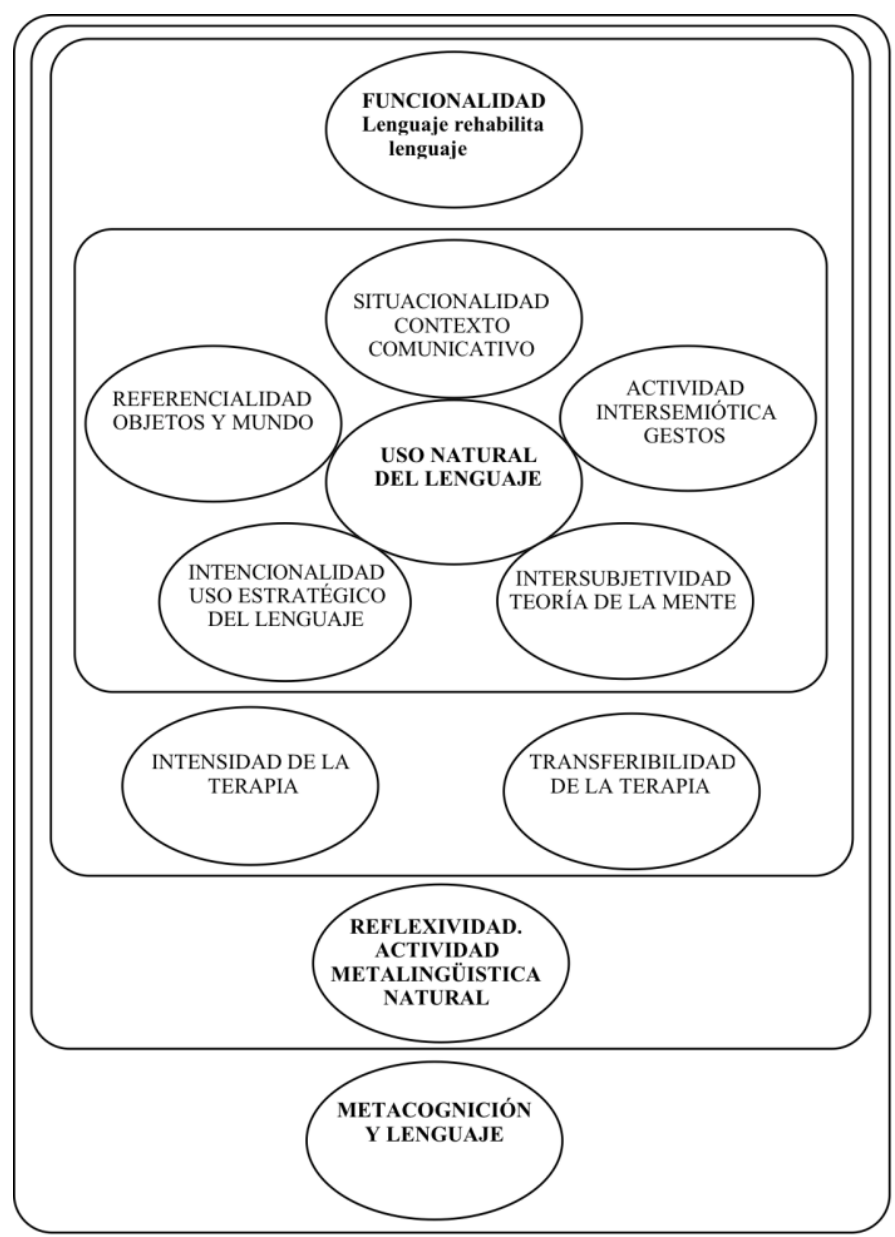

Figura 1: Paradigma pragmático-funcional en la rehabilitación de la afasia

Estrato 1: Dimensión pragmática de los hechos lingüisticos. En el dominio central de este paradigma terapéutico se sitúa el uso natural del lenguaje. El uso del lenguaje en entornos naturales debe considerarse el objetivo último de la actividad terapéutica, pero también la fuente primaria en la que se fundamentan las herramientas terapéuticas. Por uso natural del lenguaje se entiende uso contextualizado, esto es, el que puede ser observado de manera paradigmática en la práctica conversacional. Los factores contextuales 
relevantes para el uso natural del lenguaje incluyen al menos: la situacionalidad, la referencialidad, la intencionalidad, la intersubjetividad y la actividad intersemiótica. A continuación, se ofrece una breve descripción de estos factores:

Situacionalidad. Se debe proponer un contexto o situación comunicativa específica para estimular la adopción de estrategias también específicas en el uso del lenguaje. Cuando no son accesibles situaciones comunicativas reales, las actividades de juego de roles pueden aproximarse a ellas. Referencialidad. Los objetos referidos deben formar parte de situaciones cotidianas vividas por el paciente. Hablar de experiencias personales asociadas a los objetos referidos tiene un gran valor terapéutico, especialmente en lo que se refiere a la transferibilidad de los logros clínicos. Las experiencias personales estimulan los factores motivacionales y emocionales involucrados en la producción y la comprensión del lenguaje.

Actividad intersemiótica. La actividad intersemiótica debe incluirse en el programa terapéutico. La entonación y los gestos fónicos merecen una atención especial, al igual que los gestos kinésicos. El contexto conversacional proporciona también la manifestación paradigmática de esta actividad intersemiótica. De hecho, según Rose y Douglas (2003): "Natural conversational settings enlarge in a very significant way the scope of gesturing abilities that formal limb apraxia tests can initially establish" (apud Hernández-Sacristán, Rosell-Clari y MacDonald, 2011: 43)

Intencionalidad/Papel activo del paciente. El terapeuta ofrece opciones al paciente para su actividad verbal, con el fin de fomentar un uso estratégico y libre del lenguaje guiado por propósitos comunicativos personales. Al igual que en otros procesos de aprendizaje o re-aprendizaje, la terapia del lenguaje requiere la participación activa del paciente como un factor crucial para el éxito de la intervención. Por implicación activa entendemos la capacidad de elegir entre diferentes formas de decir las cosas, en lugar de repetir frases estándar o predecibles. La participación activa estimula los componentes experienciales, emocionales y motivacionales del uso del lenguaje.

Intersubjetividad/Papel del Interlocutor. El papel del interlocutor puede ser simulado por medio de situaciones de juego de roles y está concebido para estimular la actividad de una teoría de la mente o el trabajo intersubjetivo general. La actividad cooperativa opera en la "zona de desarrollo próximo” de los procesos de aprendizaje, según Vygotsky (1978). Es posible extrapolar esta idea a los procesos de reaprendizaje en un programa de terapia del lenguaje.

Estrato 2: Funcionalidad y trabajo terapéutico. Una vez que hemos establecido el uso del lenguaje en contexto como nuestro punto de partida y como objetivo final de un programa terapéutico, el segundo estrato de un 
paradigma pragmático-funcional puede ser identificado con el trabajo terapéutico que mejora los componentes relacionales del lenguaje. Esto significa asumir una perspectiva funcional específica. La intensidad de la terapia y la transferibilidad de los logros clínicos son co-factores adicionales en esta perspectiva funcional específica. Los componentes del estrato 2 pueden describirse de la siguiente manera:

Funcionalidad. Asumiendo la idea de que "el lenguaje rehabilita el lenguaje", el terapeuta explota las conexiones funcionales entre los dominios lingüísticos con fines terapéuticos. Por ejemplo, podemos utilizar circunloquios en el caso de pérdida de elementos léxicos, y otras relaciones léxicas como las de antonimia, sinonimia, homonimia, etc. El entorno funcional de una unidad o componente lingüístico específico admite diferentes tipos de concreción.

Intensidad del trabajo terapéutico. La relevancia de la intensidad como factor de eficacia del trabajo terapéutico puede explicarse en términos de una condición general de reaprendizaje necesaria para una activación coordinada de los componentes relacionales del lenguaje. Obviamente, este factor debe adaptarse a las condiciones fisicas y neuropsicológicas particulares del paciente. La participación efectiva de la memoria de trabajo y otras funciones ejecutivas en el proceso de rehabilitación lingüística depende en cierta medida de la intensidad del trabajo terapéutico.

Transferibilidad del trabajo terapéutico. Nos referimos aquí a características de la terapia que ayudan a transferir eficazmente resultados a situaciones comunicativas cotidianas o, en general, a situaciones comunicativas fuera de la sesión clínica. La transferibilidad implica la analogía entre situaciones comunicativas y la similitud en las estrategias de procesamiento del lenguaje.

Estrato 3: Reflexividad. Actividad metalingüistica natural: Se entiende por reflexividad (Hockett, 1960) la capacidad del usuario de la lengua de introducir una distancia mental con respecto a su propia conducta verbal, asignando así al lenguaje el estatus de un objeto de percepción diferenciado. La reflexividad puede describirse como habilidad metalingüística natural, crucial para el desarrollo del lenguaje y para el uso estratégico y funcional del lenguaje. Las actividades de monitorización son las manifestaciones más obvias de esta habilidad, pero la reflexividad también es necesaria para explicar otras técnicas características del uso natural del lenguaje según lo evaluado en el protocolo MetAphAs.

Estrato 4: Actividad metacognitiva relacionada con el lenguaje. Existen dos maneras de especificar las relaciones entre el lenguaje y la actividad metacognitiva o el funcionamiento ejecutivo. Por un lado, el lenguaje es un instrumento que contribuye al desarrollo de la actividad metacognitiva. En este sentido, la terapia del lenguaje puede ser útil para mejorar la memoria, la 
atención, la inhibición y otras funciones ejecutivas. Por otro lado, el lenguaje puede ser considerado como un objeto de la actividad metacognitiva. En este sentido, un tratamiento específico inicial de las funciones ejecutivas puede ser útil para la terapia del lenguaje.

\section{Conclusiones}

Como se ha señalado anteriormente, el paradigma pragmático-funcional define un plan de trabajo heurístico en el que los componentes no pueden concebirse como compartimentos estancos y no es necesario ordenarlos secuencialmente. La selección de un dominio de trabajo específico (por ejemplo, la actividad intersemiótica que liga gestualidad y lenguaje) puede producir un beneficio en otros dominios involucrados en este paradigma (situacionalidad, intersubjetividad, intencionalidad, funcionalidad, etc.) en diferentes grados. Al igual que con otros planes heurísticos, se requiere cierto grado de creatividad para seleccionar las tareas más adecuadas y su secuencia a fin de poner en marcha un programa terapéutico específico. Esto supone también una implicación activa del terapeuta, que no es un sujeto que aplica mecánicamente un instrumental previamente diseñado.

El paradigma pragmático-funcional asume la idea de que cada paciente requiere ejercicios de rehabilitación adaptados a sus necesidades, intereses y motivaciones comunicativas particulares, y que tengan en cuenta las capacidades cognitivas preservadas. Siguiendo la pauta establecida en RosellClari y Hernández-Sacristán (2017), el programa de rehabilitación proporciona un contexto en el que el paciente puede tomar sus propias decisiones sobre qué, cómo y cuándo comunicar. Tiene un papel activo en la especificación del tratamiento, el cual puede ser modificado de acuerdo a las habilidades y destrezas puestas en juego por el paciente que realiza las tareas de rehabilitación. Los ejercicios se adaptan en función del grado de dificultad, el tipo de tarea y el objetivo específico que se plantea y que debe ser siempre acorde con las necesidades y expectativas del paciente. El proceso de terapia involucra al paciente en situaciones comunicativas naturales o situaciones que evocan un uso natural del lenguaje. Los procedimientos de la terapia se centran en la descripción y el análisis de la situación (contexto), en los sentimientos, intenciones y pensamientos de los participantes en esta situación (componente personal), y en lo que estos participantes probablemente están diciendo, cómo lo están diciendo (incluyendo gestos y entonación), cuáles pueden ser sus intenciones, qué tipo de errores podrian cometer y cómo podrían hacer frente a sus disfuncionalidades lingüísticas y comunicativas. En conclusión, una perspectiva funcional implica considerar el trabajo terapéutico lingüístico como algo que siempre opera en las redes multidimensionales asociadas a objetivos terapéuticos específicos. 


\section{REFERENCIAS}

ALI, M. et al. (2013): "More outcomes than trials: A call for consistent data collection across stroke rehabilitation trials", International Journal of Stroke, 8, pp. $18-24$

ARMSTRONG, E. \& FERGUSON, A. (2010): "Language, meaning, context, and functional communication", Aphasiology, 24, pp. 480-496.

BARKLEY, R. A. (1997): "Behavioural inhibition, sustained attention, and executive functions: Constructing a unifying theory of ADHD”, Psychological Bulletin, 121, pp. 65-94.

BRADY M. C. et al. (2012): "Speech and language therapy for aphasia following stroke", Cochrane Database of Systematic Reviews, 5, Art. No.: CD000425.

BRADY, M. C. et al. (2014): “Time for a step change? Improving the efficiency, relevance, reliability, validity and transparency of aphasia rehabilitation research through core outcome measures, a common data set and improved reporting criteria", Aphasiology, 28, pp. 13851392.

CARTER, A.; CONNOR, L. y DROMERICK, A. (2010): "Rehabilitation after stroke: Current state of science", Current Neurology and Neuroscience Reports, 10, pp. 158-166.

FRANCIS, D. R.; CLARK, N. \& HUMPHREY, G. W. (2002): "Circumlocution-induced naming. A treatment for effecting generalisation in anomia?", Aphasiology, 16, pp. 234-259.

FRANKEL, T. \& PENN, C. (2007): "Perseveration and conversation in TBI: Response to pharmacological intervention", Aphasiology, 21, pp. 1039-1078.
FRANKEL, T.; PENN, C. \& ORMONDBROWN, D. (2007): "Executive dysfunction as an explanatory basis for conversation symptoms of aphasia: A pilot study." Aphasiology, 21, pp. 814-828.

GALLARDO PAÚLS, B. y HERNÁNDEZ SACRISTÁN, C. (2013): Lingüística clinica. Un enfoque funcional sobre las alteraciones del lenguaje, Madrid: Arco Libros.

GOODWIN, C. (1995): "Co-constructing meaning in conversation with an aphasic man", $R e$ search on Language and Social Interaction, 28, pp. 233-260.

GOODWIN, C. (2000): "Pointing and the collaborative construction of meaning in aphasia", Proceedings of the 7th Annual Symposium About Language and Society, Austin: University of Texas, pp. 67-76.

HALLIDAY, M. A. K. (1978): Language as a Social Semiotic: Social Interpretation of Language and Meaning, London: Edward Arnold

HELM-ESTABROOKS, N. \& ALBERT, M. L. (1991): Manual of Aphasia Therapy, Austin TX: PRO-ED.

HELM-ESTABROOKS, N. \& ALBERT, M. L. (2003): Manual of Aphasia and Aphasia Therapy, Austin TX: PRO-ED.

HERNÁNDEZ SACRISTÁN, C. (2019): "Anomia: Una perspectiva enaccionista sobre la incapacidad de acceso a las unidades léxicas", Pruñonosa-Tomás, M. (ed.): Lenguaje, paisaje lingüistico y enacción, Valencia, Tirant Humanidades, pp. 261-275.

HERNÁNDEZ-SACRISTÁN, C.; ROSELL-CLARI, V. \& MACDONALD, J. E. (2011): "Proximal and distal. Rethinking linguistic form and use for clinical 
purposes", Clinical Linguistics $\&$ Phonetics, 25, pp. 37-52.

HERNÁNDEZ-SACRISTÁN, C. et al. (2012): “On natural metalinguistic abilities in aphasia. A preliminary study", Aphasiology, 26, pp. 199-219.

HOCKETT, C. (1960): "The origin of speech", Scientific American, 203, pp. 88-96.

HOLLAND, A. (1979): "Some practical considerations in aphasia rehabilitation", Sullivan, M. y Kommers, M. (eds.): Rationale for adult aphasia therapy, Omaha: University of Nebraska Medical Center, pp. 167-180.

HOLLAND, A. (1980): Communicative Abilities in Daily Living: A test of functional communication for adults, Baltimore: University Park Press.

HOLLAND, A. (1991): "Pragmatic aspects of intervention in aphasia", Journal of Neurolinguistics, 6, pp. 197-211.

JOHNSON, M. L. et al. (2014): “An Enhanced Protocol for ConstraintInduced Aphasia Therapy II: A Case Series", American Journal of Speech-Language Pathology, 23, pp. 60-72.

KAGAN, A. (1998): "Supported conversation for adults with aphasia: methods and resources for training conversational partners", Aphasiology, 12, pp. 816-830.

KAGAN, A. et al. (2001): "Training volunteers as conversation partners using Supported conversation for adults with aphasia (SCA): A controlled study", Journal of Speech, Language, and Hearing Research, 44, pp. 624-638.

MCNEIL, M. R. et al. (1995): "Behavioral and pharmacological treatment of lexical-semantic deficits in a single patient with primary progressive aphasia", American Journal of Speech
Language Pathology, 4, pp. 7687.

PENN, C. et al. (2010): "Executive function and conversational strategies in bilingual aphasia", Aphasiology, 24 (2), pp. 288308.

PERKINS, M. (2000): “The scope of pragmatic disability. A cognitive approach", Müller, N. (ed): Pragmatics in Speech and Language Pathology: Studies in Clinical Applications, Amsterdam: John Benjamins, pp. 628.

ROSE, M. (2006): "The utility of arm and hand gestures in the treatment of aphasia", Advances in Speech Language Pathology, 8, pp. 92-109.

ROSE, M. \& DOUGLAS, J. (2003): "Limb apraxia, pantomime, and lexical gesture in aphasic speakers: preliminary findings", Aphasiology, 17, pp. 453464.

ROSELL-CLARI，V. y HERNÁNDEZSACRISTÁN, C. (2014): MetAphAs. Protocolo de Exploración de Habilidades Metalingüisticas en la Afasia, Valencia: NauLlibres.

ROSELL-CLARI, V. \& HERNÁNDEZSACRISTÁN, C. (2017): "Anomia rehabilitation viewed from a pragmatic-functional paradigm. A case study", Revista de Investigación en Logopedia, 7, pp. 47-70.

ROSELL-CLARI, V. \& HERNÁNDEZSACRISTÁN, C. (2018a): MetAphAs. Metalanguage in Aphasia Assessment, Valencia: NauLlibres (Libro electrónico en Google books).

ROSELL-CLARI, V. \& HERNÁNDEZSACRISTÁN, C. (2018b): "Aphasiarehabilitationfrom a pragmatic-functionalparadigm. A comparative study of two patients with different perfor- 
mance profiles in natural metalinguistic tasks", Aphasiology, 32 (S1), pp. 50-51.

ROSELL-CLARI, V. \& VALLES-GONZÁLEZ, B. (2016): “Theory of Mind (ToM) and language: stimulating metalinguistic skills in people with dementia", $R e$ vistaCoDAS, 28, pp. 252-260.

SALTER, K. L. et al. (2007): "Outcome assessment in randomized controlled trials of stroke rehabilitation", American Journal of Physical Medicine \& Rehabilitation, 86, pp. 1007-1012.

SANDT-KOENDERMAN M. E., MEULEN I. \& RIBBERS G. M. (2012): "Aphasia rehabilitation: more than treating the language disorder", Archives of Physical Medicine and Rehabilitation, 93 (1), pp. 1-3.

SARNO, M. T. (1969): The Functional Communication Profile: Manual of directions, New York: Institute of Rehabilitation Medicine.

SIMMONS-MACKIE, N. et al. (2010): "Communication Partner Training in Aphasia: A Systematic
Review", Archives of Physical Medicine and Rehabilitation, 91, pp. 1814-1837.

SPENCER, K. A. et al. (2000): "Examining the facilitative effects of rhyme in a patient with output lexical damage", Aphasiology, 14 , pp. 567-584.

TOMASELLO, M. (2003): Constructing a Language. A Usage-Based Theory of Language Acquisition, Cambridge, Mass.: Harvard University Press.

VYGOTSKY, L. S. (1978): Mind in Society: The Development of Higher Psychological Processes, Cambridge, MA: Cambridge University Press.

WILKINSON, R. \& WIELAERT, S. (2012): "Rehabilitation targeted at everyday communication: Can we change the talk of people with aphasia and their significant others within conversation?", Archives of Physical Medicine and Rehabilitation, 93 (1), pp. 70-76. 